\title{
Fusion bonding of carbon fabric reinforced polyphenylene sulphide
}

\author{
De Baere I. ', Van Paepegem W. and Degrieck J. \\ Ghent University,Department of Materials Science and Engineering \\ Sint-Pietersnieuwstraat 41, B-9000 Gent, Belgium
}

\begin{abstract}
In recent years, there is a growing interest in joining techniques for thermoplastic composites as an alternative to adhesive bonding. In this manuscript, a fusion bonding process called hot-tool welding is investigated for this purpose and the used material is a carbon fabric reinforced polyphenylene sulphide.

The quality of the welds is experimentally assessed using a short three-point bending setup, which has an interesting distribution of interlaminar shear stresses. It can be concluded that although the hot-tool welding process shows high short-beam strengths, it has some drawbacks. Therefore, a design of an infrared welding setup is presented.
\end{abstract}

\section{Introduction}

An ideal structure would be designed without joints, since joints are potential sources of weakness and additional weight. In practice, however, the maximum size of a component is generally limited by the manufacturing processes. Moreover, demands such as inspection, accessibility, repair and of course transportation and assembly result in the fact that load-bearing joints cannot be avoided. This fact does not change when designing with fibre-reinforced composites; joints can be drastically reduced, but they will always be a part of a structure. Adhesive bonding is inherently preferable to mechanical fastening because of the continuous connection, avoiding large stress concentrations induced by each discrete fastener hole. However, extensive surface preparation and long curing times make adhesive bonding labour intensive. Moreover, the need for recyclability incites more and more manufacturers to choose materials and bonding systems which allow for recycling, excluding most thermosetting composites and adhesives.

Therefore, in recent years, the interest is growing in welding processes for thermoplastic composites, since (i) thermoplastics are difficult to bond because of their chemical inertness and (ii) the welding processes can reduce overall manufacturing cost and are expected to replace traditional assembly methods, such as adhesive and solvent bonding, mechanical fastening and co-consolidation bonding [1]. The fusion bonding of pure thermoplastics is already a well known and commonly applied production process, but the process parameters cannot be extrapolated to the welding of fibre-reinforced thermoplastics, since the reinforcement has a large influence: the material is no longer isotropic, head conduction is influenced ... In general, these fusion bonding techniques can be categorised in three groups [1]: (i) frictional welding, including ultrasonic welding [2, 3]; (ii) electromagnetic welding, including resistance welding $[4,5,6$,$] and induction welding [7,8]$ and (iii) thermal welding, including infrared welding [9] and hot-tool welding [10].

${ }^{\text {a }}$ E-mail: Ives.DeBaere@UGent.be 
In this manuscript, the hot-tool welding process is considered as fusion bonding technique for a carbon fibre reinforced polyphenylene sulphide. This technique has some interesting advantages, for instance: dissimilar thermoplastics can be welded, the temperature of the molten interfaces can be accurately controlled, surface inaccuracies can be taken into account during the process and it can handle complex geometries [1]. Furthermore, it is a relatively cheap process, since it does not require expensive machinery, as is the case for friction- and ultrasonic welding [1].

To evaluate the quality of the weld, a short-beam bending test, as described in the ASTM D2344/D 2344M 'Standard Test Method for Short-Beam Strength of Polymer Matrix Composite Materials and Their Laminates' is considered. This test has the advantage that it requires a lot less material than for the lap shear or mode I DCB test and it is a very simple test; force and displacement already give plenty of information. Also, this setup induces an interesting stress distribution in the bond, as was seen by finite element modelling in ABAQUS ${ }^{\mathrm{TM}}$ [10].

\section{Materials and Methods}

\subsection{Composite Material}

The material used for the experiments was a 5-harness satin-weave carbon fabric-reinforced polyphenylene sulphide (PPS). The carbon PPS plates were hot pressed, one stacking sequence was used for this study, namely $\left[\left(0^{\circ}, 90^{\circ}\right)\right]_{2 \mathrm{~s}}$ where $\left(0^{\circ}, 90^{\circ}\right)$ represents one layer of fabric.

The test coupons were sawn with a water-cooled diamond saw; the dimensions of the specimens are shown in Fig.ure 1. The dimensions of the welded specimen are chosen so that from each weld, three bending coupons can be cut.

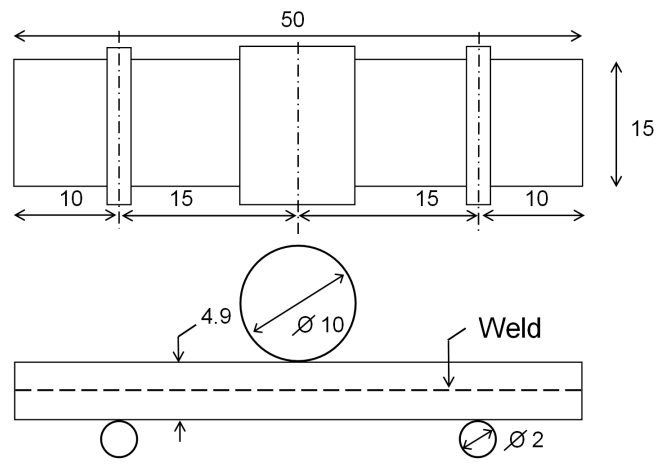

(a) Bending specimen

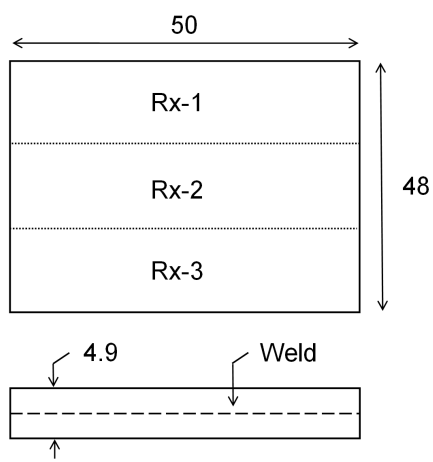

(b) Welded specimen

Fig. 1 Dimensions of the used specimens in millimetres.

To evaluate the strength, the short beam strength, as mentioned in the ASTM D2344/D 2344M 'Standard Test Method for Short-Beam Strength of Polymer Matrix Composite Materials and Their Laminates' is calculated using Equation 1:

$$
\tau^{s b s}=0.75 \frac{P_{m}}{b h} \quad[\mathrm{MPa}]
$$

where $\tau^{\text {sbs }}$ is the short-beam strength [MPa], $\mathrm{P}_{\mathrm{m}}$ is the maximum load observed during the test $[\mathrm{N}]$, $\mathrm{b}$ is the width of the specimen [mm] and $\mathrm{h}$ is the height of the specimen [mm].

\subsection{Equipment}

All bending tests were performed on an electromechanical INSTRON 5800R tensile testing machine with a FastTrack 8800 digital controller and a load cell of $\pm 10 \mathrm{kN}$. The quasi-static bending tests were displacement-controlled with a speed of $2 \mathrm{~mm} / \mathrm{min}$. For the registration of the data, a 


\section{$14^{\text {th }}$ International Conference on Experimental Mechanics}

combination of a National Instruments NI-USB-6251 data acquisition card and the SCB-68 pin shielded connecter were used. The load and displacement, given by the FastTrack controller, as well as the temperature from the thermocouple were sampled on the same time basis.

\section{Experiments and discussion}

\subsection{Welding Process}

The fusion bonding process of choice is the 'hot-tool welding', of which the principle is illustrated in Figure 2 (a). The two surfaces to be welded are pushed against a heating element (step 1) and once the temperature is high enough, the two parts are pressed against one another with sufficient force (step 2). The 'hot-tool' is shown in Figure 2 (b). The surfaces of the parts to be welded make contact with the top and bottom contact plate respectively and the heat is generated using a heating resistor. A thermocouple is embedded near the surface of the hot-tool to control the temperature of the unit. The power of the resistor is controlled with the separate control unit, which takes the temperature into account. With this device, an area of $100 \mathrm{~mm} \times 100 \mathrm{~mm}$ can be heated. For this research, an extra plate was added on top of the welding device, so that a contact area of $50 \mathrm{~mm}$ wide can be heated, in order to produce the specimens illustrated in Figure 1 (b).

One of the disadvantages of hot-tool welding is that some thermoplastic material remains on the hot-tool. By using this plate, it is easier to keep the hot-tool clean and a number of films can be wrapped around this plate to avoid the sticking. Both TEFLON and KAPTON were assessed for this purpose, since these materials survive at the melting temperature of PPS, which is $280{ }^{\circ} \mathrm{C}$, but eventually, the best method was using no film at all and making contact between the steel plate and the composite. PPS always stuck to the film, but the film itself made the removing of the hot-tool prior to the consolidation phase a lot more cumbersome.

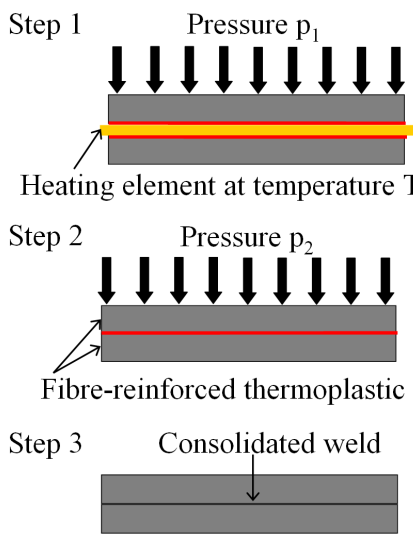

(a) Principle of hot-tool welding

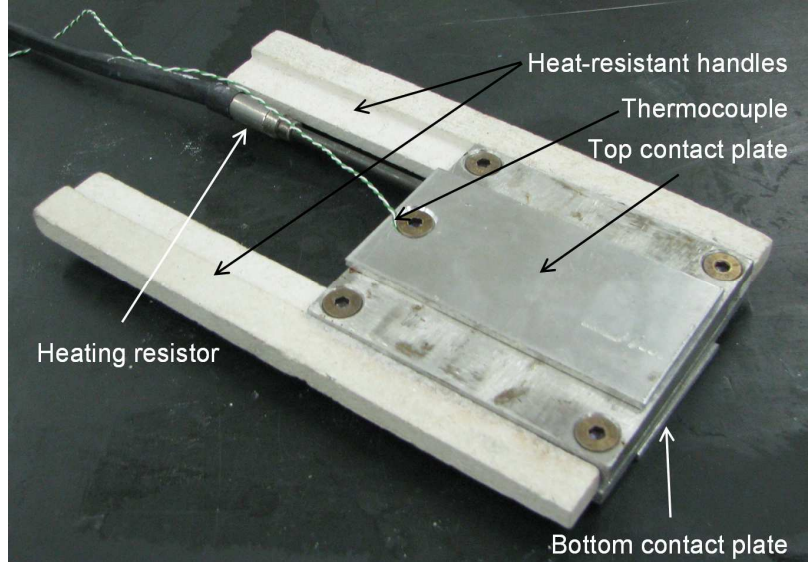

(b) The actual setup

Fig. 2 The 'hot-tool' welding process and device.

During the welding phase, one can choose to hold the contact pressure constant or to press down until the weld has a certain thickness. For this study, the contact pressure was increased to the desired value and then the displacement was kept constant. Figure 3 illustrates the evolution of the contact pressure and the temperature during welding. The specimen was heated for about 80 seconds at an average temperature of $305{ }^{\circ} \mathrm{C}$. The temperature was set at $310{ }^{\circ} \mathrm{C}$ but because of the high thermal conductivity of carbon, a lot of heat was dissipated to the surroundings and to the tensile machine. Since the temperature of the hot-tool is monitored, only the evolution of the temperature during the heating phase is relevant. Since the embedding of a thermocouple in the weld poses some difficulty, there is no information present on the evolution of the temperature during the consolidation. 
There is some scatter on the contact pressure, since it is quite difficult to maintain a constant load, even in load-controlled mode. The decrease in contact pressure during consolidation is due to the fact that the pressure pushes the liquid PPS out of the weld. The specimen is removed once the weld is fully solidified.

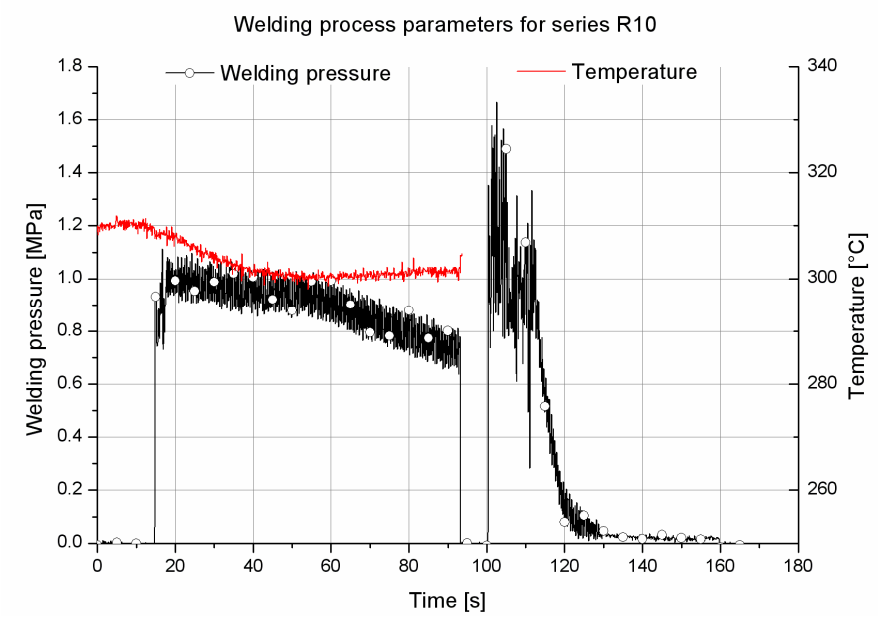

Fig. 3 Illustration of the temperature and the welding pressure during the welding process

\subsection{Bending Experiments}

In order to have some sort of reference value, first, some specimens were adhesively bonded with adhesives that gave good results in the past [11]. The results are shown in Figure 4, together with two bending tests on not bonded specimens. These are added to evaluate the bending stiffness when the bond has failed completely. For the adhesively bonded specimens, it can be clearly seen that once a load level around 1800 to $2000 \mathrm{~N}$ is reached, corresponding to a short-beam strength of $20.4 \mathrm{MPa}$, a very brittle interlaminar failure occurs and the force-displacement curve then follows the same trend as the curve from the unbonded specimens. As such, the quality of the weld can also be assessed by the bending stiffens. The load-displacement of the failed bonded specimens lies a little higher than the not bonded specimen, but this is due to the fact that only half of the bond failed, between one outer support and the centre support.

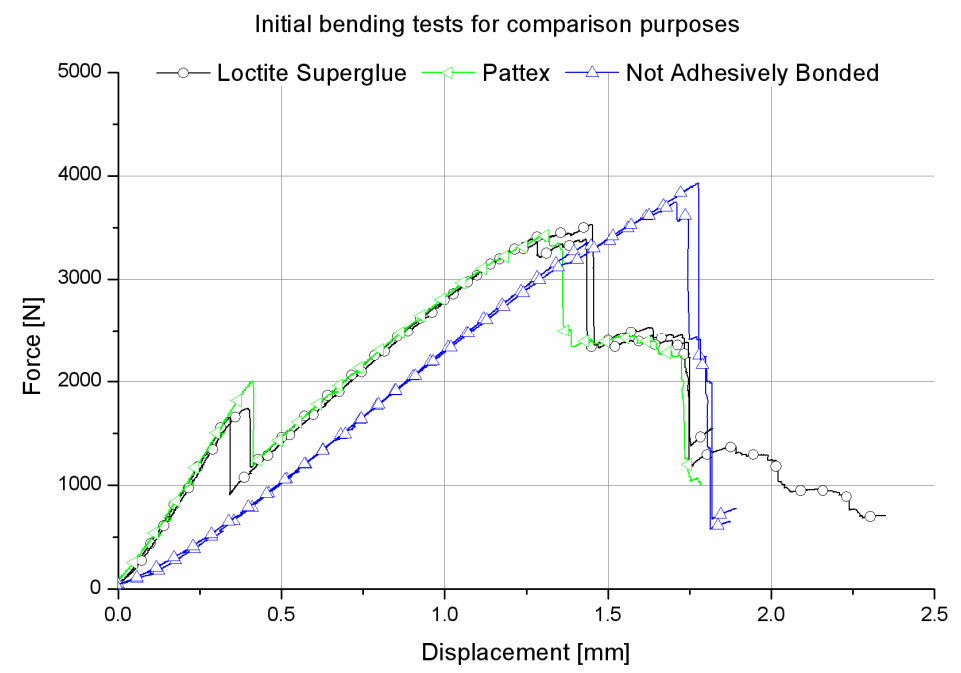

Fig. 4 Bending experiments on adhesively bonded specimens 


\section{$14^{\text {th }}$ International Conference on Experimental Mechanics}

A first series of tests were conducted to evaluate the reproducibility of the quality of the welds of one welded specimen, as illustrated in Figure 1 (b). It could be concluded that there is a good reproducibility, especially with respect to the bending stiffness.

Figure 5 shows several bending experiments on specimens from different welding processes. As can be seen, the reproducibility of the bending stiffness is high. With respect to the failure force, this value of course depends on the welding parameters, but even for a not so successful weld (R4-3) the failure force is still significantly higher than for the adhesively bonded specimens. It can also be noticed that most specimens tend to follow the curve of two separate, not bonded specimens after initial failure, meaning that failure is still quite brittle, although specimen R14-1 shows a very progressive failure.

Finally, Table 1 shows an overview of the welding parameters for the experiments discussed in this paper. The contact pressure during consolidation was always the same as the contact pressure during heating. This pressure was chosen close to $1 \mathrm{MPa}$, since this is also the consolidation pressure during the hot pressing of the composite plates. In earlier experiments, tests were done with contact pressures up to $3 \mathrm{MPa}$, but with results inferior to the ones shown here, because al liquid PPS was pushed out of the bond. Therefore, these experiments and settings are not discussed in this manuscript.

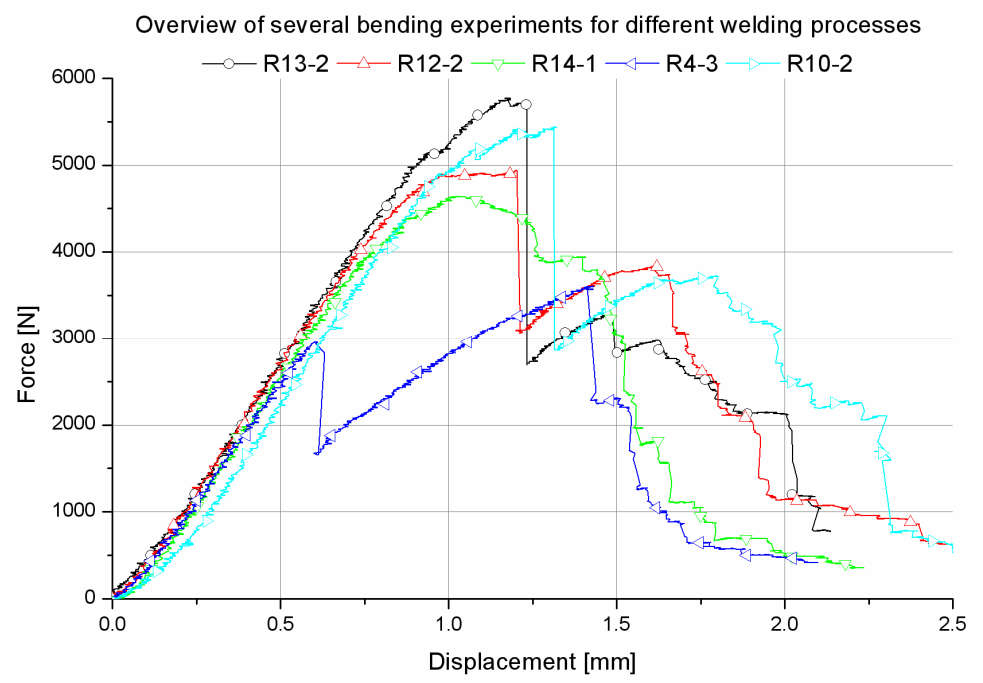

Fig. 5 Force-displacement curves for specimens from different welding processes

With respect to the temperature, the melting point of PPS is $280{ }^{\circ} \mathrm{C}$, so temperatures lower were not considered. A temperature higher than $310^{\circ} \mathrm{C}$ was too high; on some locations of the welded surface, the PPS started to burn whereas on other locations (for the same weld) the PPS had not even melted. As such, the two specimens could be joined together, but there was no continuous weld and the strength was, of course, inferior. For the successful welds (R10, R12, R13 and R14), an average short-beam strength of $52.4 \mathrm{MPa}$ is achieved.

Table 1 Overview of the welding parameters for the experiments shown in this paper, together with the shortbeam strength (Equation 1)

\begin{tabular}{ccccc}
\hline Process & Contact pressure $[\mathrm{MPa}]$ & Temperature $\left[{ }^{\circ} \mathrm{C}\right]$ & Heating time $[\mathrm{s}]$ & $\tau^{\mathrm{sbs}}[\mathrm{MPa}]$ \\
$\mathrm{R} 4$ & 0.9 & 290 & 60 & $30 \pm 5.2$ \\
$\mathrm{R} 10$ & 0.9 & 300 & 75 & $55.0 \pm 0.5$ \\
$\mathrm{R} 12$ & 1 & 310 & 20 & $46.9 \pm 3.5$ \\
$\mathrm{R} 13$ & 1 & 290 & 100 & $54.8 \pm 4.2$ \\
$\mathrm{R} 14$ & 1 & 300 & 70 & $52.7 \pm 5.3$ \\
\hline
\end{tabular}




\section{Design of an infrared welding device}

Although the short-beam strengths, achieve with the hot-tool device, are very promising for further research, the presented setup has some drawbacks. The most important one is the fact that the liquid matrix sticks to the hot-tool upon removal, meaning that an unknown but significant quantity of welding material is lost. Furthermore, although theoretically possible, it is difficult to add extra thermoplastic to the weld, since after the removal of the hot-tool, the molten specimens solidify very quickly and in most cases, insufficient heat remains present to melt the added thermoplastic and form a strong bond. A second drawback is the fact that the removal of the hot-tool is quite cumbersome because of the sticking thermoplastic, making the setup difficult to automate. To overcome these drawbacks, infrared welding will be considered. With this setup, heat is generated using strong infrared lights which are placed between the two specimens, without making contact , solving the sticking problem. After melting, the lamps are removed and the two coupons are pushed together using a set of pneumatic actuators. The total setup, including power electronics and pneumatics, is shown in Figure 6 (a). Figure 6 (b) shows a detail of the infrared lamps, mounted in the movable frame.

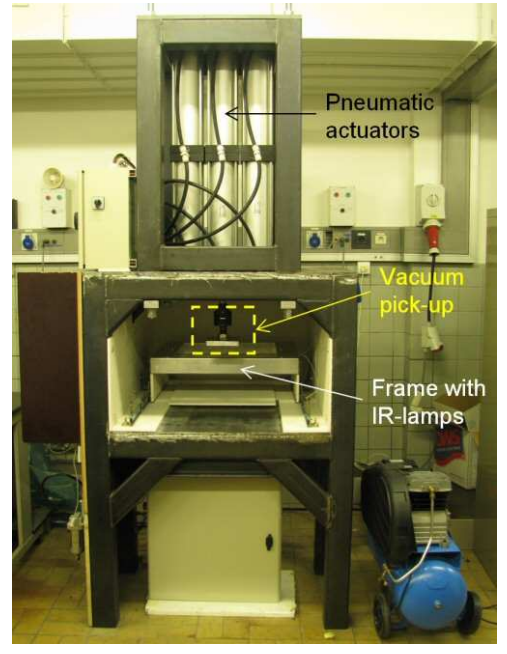

(a) Total setup

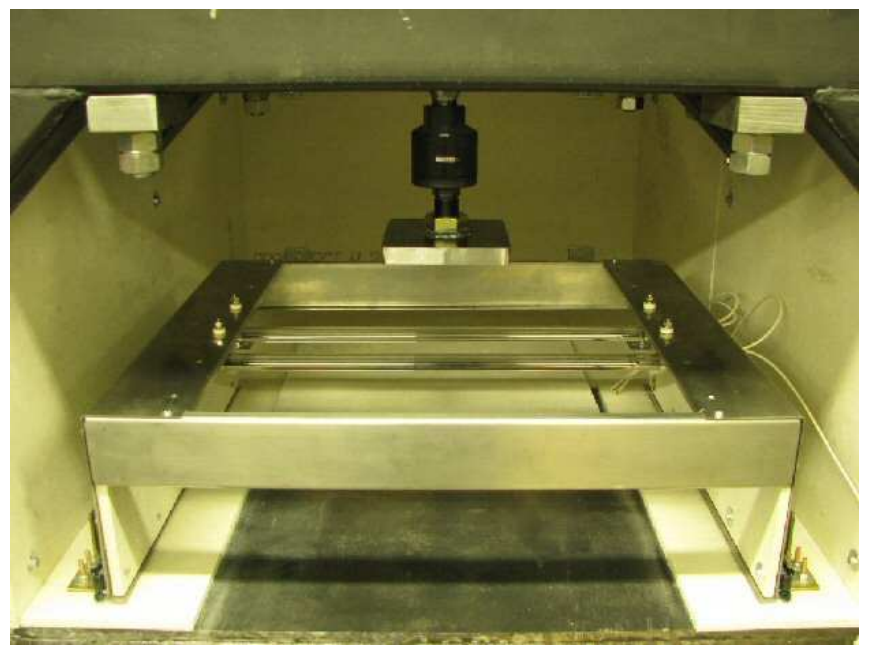

(b) IR-lights within frame

Fig. 6 The infrared welding setup

The pneumatic actuators operate at 10 bar, yielding a maximum force of $22.5 \mathrm{kN}$, corresponding to a welding pressure (welding pressure) of $1 \mathrm{MPa}$, which should be sufficient, considering the results from the hot-tool process mentioned earlier. The setup is designed for nine pneumatic actuators; currently, three are mounted, meaning that an area of $150 \mathrm{~mm}$ x $450 \mathrm{~mm}$ can be welded. With the nine actuators, an area of $450 \mathrm{~mm} \times 450 \mathrm{~mm}$ can be heated and welded.

Underneath the actuator, a vacuum pick-up system is mounted to hold the top plate over the infrared light frame during heating. The bottom plate simply rests on the frame.

The infrared lights use a carbon filament and generate a power of $4000 \mathrm{~W}$ each. Six lamps can be mounted in the frame (Figure 6 (b) shows the two central lights) and they are controlled two by two. A separate control system continuously monitors the temperature of the specimens, using a noncontact temperature sensor and controls the power sent to the IR-set.

\section{Conclusions}

The hot-tool welding process is suited for welding the carbon-fabric reinforced polyphenylene sulphide considered for this research. The interlaminar strength in a short three-point bending test is significantly higher than for adhesively bonded specimens. 
For the welding parameters, a temperature between $295^{\circ} \mathrm{C}$ and $305^{\circ} \mathrm{C}$ in combination with a contact pressure of $1 \mathrm{MPa}$ for about 60 seconds shows best results. Other temperatures resulted in either no melting or burning of the PPS. Lower contact pressures resulted in bad consolidation and higher pressure pushed out all PPS, so no bond could be formed. The time interval is not so narrow, but in general it may be concluded that within the specified range of temperature and pressure, the quality of the weld is mostly dependent on the experience of the operator. For the successful welds, an average short-beam strength of $52.4 \mathrm{MPa}$ was achieved.

Despite the high short-beam strength achieved, the fact that a lot of PPS stuck to the hot-tool is a serious drawback, since it removes an unknown but significant quantity of welding material out of the weld. Also, the current process is difficult to automate, due to the cumbersome removal of the hot-tool. Therefore, an infrared welding setup has been designed, which no longer has these drawbacks.

\section{ACKNOWLEDGMENTS}

The authors would like to thank Ten Cate Advanced Composites for supplying the material and they gratefully acknowledge the finance through a grant of the Fund for Scientific Research - Flanders (F.W.O.). 


\section{References}

1. Yousefpour A, Hojjati M, Immarigeon JP, Fusion bonding/welding of thermoplastic composites. Journal of Thermoplastic Composite Materials 17 (4): 303-341 (2004).

2. Gutnik VG, Gorbach NV, Dashkov AV, Some characteristics of ultrasonic welding of polymers. Fibre Chemistry 34 (6): 426-432 (2002).

3. Jandali G, Mallick PK, Vibration welding of continuous-fiber thermoplastic matrix composites. Journal of Thermoplastic Composite Materials 17 (4): 343-358 (2004).

4. Stavrov D, Bersee HEN. Resistance welding of thermoplastic composites - an overview. Composites Part A 36 (1): 39-54 (2005).

5. Ageorges C, Ye L, Hou M. Experimental investigation of the resistance welding for thermoplastic-matrix composites. Part I: heating element and heat transfer. Composites Science and Technology 60 (7): 1027-1039 (2000).

6. Ageorges C, Ye L, Hou M. Experimental investigation of the resistance welding of thermoplastic-matrix composites. Part II: optimum processing window and mechanical performance. Composites Science and Technology 60 (8): 1191-1202 (2000).

7. Kagan VA, Nichols RJ. Benefits of induction welding of reinforced thermoplastics in high performance applications. Journal of Reinforced Plastics and Composites 24 (13): 1345-1352 (2005).

8. W. Suwanwatana, S. Yarlagadda and J.W. Gillespie, Jr. Hysteresis heating based induction bonding of thermoplastic composites . Composites Science and Technology 66 (11-12): 1713-1723 (2006).

9. Lamethe JF, Beauchene P, Leger L. Polymer dynamics applied to PEEK matrix composite welding. Aerospace Science and Technology 9 (3): 233-240 (2005).

10. De Baere I., Van Paepegem W. and Degrieck J. Feasibility study of fusion bonding for carbon fabric reinforced polyphenylene sulphide by hot-tool welding. SUBMITTED to Polymer Composites.

11. De Baere I., Experimental and Numerical Study of Different Setups for Conducting and Monitoring Fatigue Experiments of Fibre-Reinforced Thermoplastics. doctoral-thesis, Ghent University, ISBN 987-90-8578-196-7, February 2008 